\title{
LA GEOMEDIACIÓN Y LOS GEOMEDIARIOS
}

\author{
Rita Lázaro Sevilla ${ }^{1}$ \\ Licenciada en Geografía
}

\section{RESUMEN}

Este artículo introduce una nueva idea en Geografía: la Geomediación, los geomediarios y los geomediadores. La geomediación se define como los sistemas de soporte a los procedimientos de mediación en planificación geográfica. Puede ser una parte de los procesos de la Cooperativa de toma de decisiones espaciales y puede plantear requerimientos específicos en el uso de mapas online. Esta idea procede de Alemania, pero también ha sido descubierta en España, destacando en este caso el papel de los geomediarios y los geomediadores como personas capaces de establecer asociaciones entre universos paralelos (AUPA).

Palabras clave: Geomediación, geomediarios, geomediadores y asociaciones entre universos paralelos (AUPA).

\section{ABSTRACT}

This article introduces a new idea in Geography: the Geomediation and the geomediaries. The Geomediation is defined as the system supporting the mediation of geographical planning procedures. GeoMediation can be part of broader CSDM (Collaborative Spatial Decision-Making) processes and may pose specific requirements on the use of online maps. Not only is made the idea from Germany, but also it is made from Spain. It is underlined the role of the geomediaries in Spain as people that can make associations between parallel universes (UPAA).

Key words: Geomediation, geomediaries, geomediators and associations between parallel universes (UPAA).

1 Este trabajo de investigación es la consecuencia del curso de doctorado sobre «Proactividad, ciberespacio y territorio»", impartido en el departamento de Geografía de la Universidad Autónoma de Madrid por Fernando Moliní Fernández en el año 2000/2001.

Fecha de recepción: 26 de junio de 2006. Fecha de aceptación: 15 de abril de 2008 . 


\section{El nacimiento de una nueva idea en Geografía}

La navegación espacial, la simulación en geografía, los sistemas de información geográfica (SIG), las imágenes aéreas y de satélite y su aplicación y difusión en Internet abren nuevas expectativas a la Geografía académica y profesional. Pero quizás una de las labores con mayor importancia del geógrafo del ahora y del que viene, junto con la de gestores del medio ambiente, consista en establecer asociaciones entre el mundo real y el mundo virtual que, valorando la sustancia del medio natural, contribuyan a mejorar el uso y organización del territorio, incluyendo el modo de vida y producción sobre el mismo.

Incluso para tareas de esta índole, existen máquinas capaces de formular hipótesis, interpretar los resultados y ajustar o descartar sus teorías para adaptarlas a los hechos, y hay una capaz de «entender el mundo». Su mecanismo básico consiste en memorizar las correlaciones más habituales entre los rasgos del mundo y usarlas para predecir el futuro. Uno de sus inventores —el ingeniero informático del Silicon Valley, Jeff Hawkins² —, está programando ordenadores para que utilicen esa idea, y está convencido de que las máquinas de ese tipo, inspiradas en la mente humana pero dotadas de la velocidad de la electrónica, pronto nos superarán en inteligencia y creatividad.

Construir ordenadores que favorezcan una comprensión mejor del mundo no es un pensamiento nuevo. Algunos científicos han investigado durante años cómo los ordenadores pueden contribuir al perfeccionamiento de la economía y de las leyes de la Tierra para beneficio de todos. Y en esa búsqueda de facilitar soluciones para todos los problemas del mundo, el ordenador se ha convertido en un modelo de la actuación humana. Tal es así que desde antes de la revolución industrial se conoce la existencia de autómatas, como el muñeco que sirve el té o la tocadora del tímpano, que por aquel entonces hacían imaginar un futuro hecho de continuos progresos, con máquinas que facilitaran todo tipo de trabajos y harían más confortables las actividades cotidianas.

El secreto de la revolución industrial estuvo en el vínculo entre ciencia y tecnología. Las posibilidades ofrecidas por la revolución industrial iban bastante más lejos del cumplimiento de las necesidades primarias. Inventores y científicos pensaban en hallazgos que hiciesen la vida cada vez más placentera y confortable, especialmente en las ciudades y, como consecuencia, se realizaron grandes progresos en el sector de la higiene pública, el alumbrado, la comunicación y el transporte.

En los últimos tiempos la llegada de la informática y la interacción de varios avances de alta tecnología — satélites espaciales, microelectrónica, fibras ópticas, rayos láser, y el ordenador - está magnificando la capacidad humana de almacenar y analizar datos, comunicar y diseminar información, y planificar decisiones para que podamos hallar mejores soluciones a los problemas del mundo

Uno de los secretos de la actual Era de la Información se encuentra en el vinculo entre Informática y Geografía, en la posibilidad de conectar a los ciudadanos del planeta con el territorio por medio de un ordenador.

La conexión entre Informática y Geografía se denomina Geomática. La Geomática, en algunos lugares del mundo llamada Geoinformática, es un término científico moderno que resulta de la unión de Ciencias de la Tierra y la Informática para expresar una integración sistémica de técnicas y metodologías de adquisición, almacenamiento, procesamiento, análisis, presentación y distribución de información geográficamente referenciada.

A nivel internacional pueden encontrarse diferentes definiciones o interpretaciones de geomática. La palabra se deriva del francés «science geomatique» y ha sido adoptado

2 El País Semanal 26/12/2004. 
internacionalmente para describir el campo de la ciencia de la información espacial. La Académie de Nice en Francia (2000) define la Geomática como la informática aplicada a la Geografía.

Todas las definiciones apuntan a la integración sistémica de algunas técnicas, tales como las relacionadas con levantamientos de datos, posicionamiento global, percepción remota y fotogrametría, cartografía automatizada y sistemas de información geográfica.

En la Era de Información se puede vivir al margen de las tecnologías, pero las consecuencias sociales de la introducción de las nuevas tecnologías van habitualmente más allá de la introducción de un nuevo artefacto que nos permitiría hacer con mayor eficacia lo mismo que hacíamos anteriormente pues suponen nuevas formas de vida. No es extraño que hoy en día nos remitan a una dirección de correo electrónico o una página de Internet si queremos o tenemos que realizar consultas o trámites. Por lo tanto, los ciudadanos del mundo se ven en la necesidad de relacionarse con los medios de algún u otro modo y son numerosas las posibilidades que supone la información planetaria para trascender la realidad y mejorarla.

No obstante, en este contexto de avance de las tecnologías de las comunicaciones y la informática así como del individualismo, a la realidad le corresponde atribuir el grado de probabilidad de que estas tecnologías prosperen además de evitar que la población sucumba totalmente a los poderes televisivos e informativos. Desde una posición que mira al exterior e interpreta el paisaje se concibe la figura del geógrafo como una persona que se encuentra entre dos mundos: el tecnológico y/o virtual y biológico y/o real, y a partir de esa posición intermedia puede ser útil al establecer asociaciones que una máquina o un ordenador no puede percibir.

La tarea de establecer asociaciones entre estos universos paralelos (AUPA) con un resultado que se manifiesta directa o indirectamente en el territorio se denomina geomediación y los encargados de esta labor geomediarios y geomediadores (Figura 1).

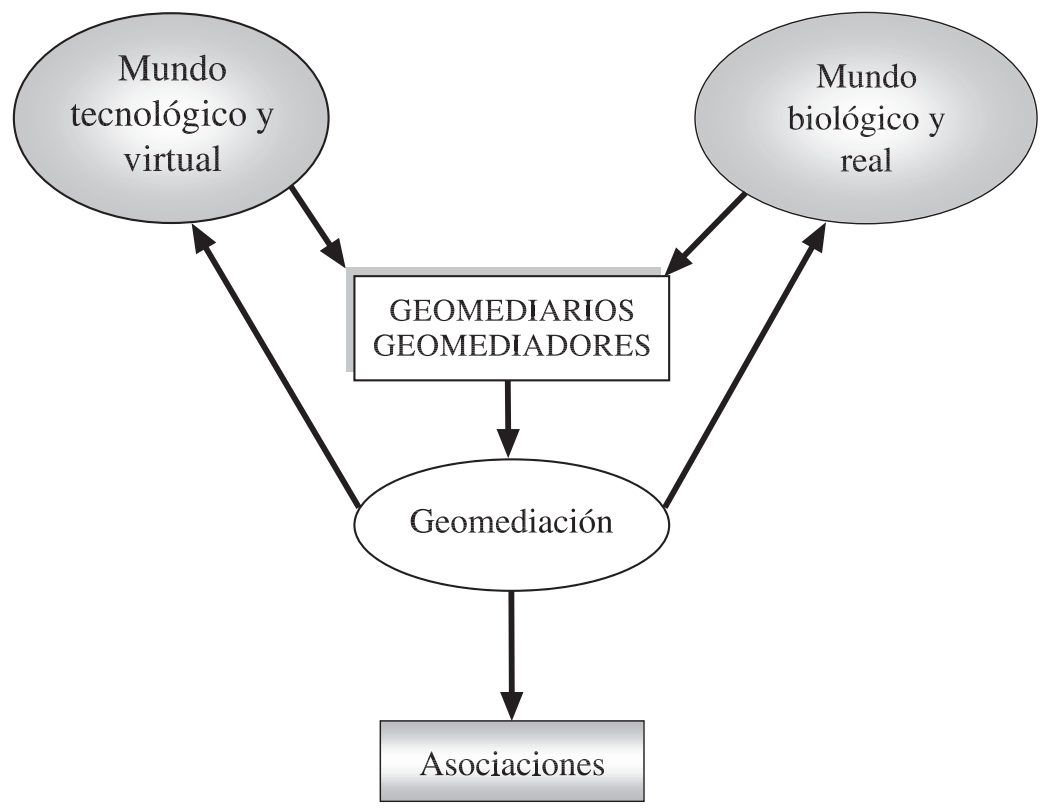

Figura 1: Proceso de geomediación. Una visión desde el Sur de Europa. Elaboración propia. 
En el proceso de Geomediación distinguimos, pues, dos universos o mundos: el tecnológico y/o virtual y el biológico y/o real. Por definición, el universo o mundo tiene diferentes acepciones pero es quizá la concepción de la Filosofía la que más se aproxima al planteamiento que postula la Geomediación.

Según la concepción filosófica, el universo es sinónimo de realidad total o de conjunto de todo lo que existe en el espacio y en el tiempo. En un sentido estricto, el Universo comprende sólo la naturaleza, y en un sentido amplio, abarca también al espíritu. Los dos términos Tierra y Humanidad expresan las dos partes integrantes del Universo.

El Universo tiene, además, un segundo aspecto para el ser humano. Este, en efecto, no sólo forma parte del Universo, sino que es un mundo dentro de otro mundo. El hombre y la mujer tienen la virtud de representarse el Universo y la necesidad de conocerlo para cumplimiento integro de sus fines. En este sentido el Universo equivale al mundo del pensamiento como un conjunto de las ideas o, más exactamente, de las clases lógicas que son tomadas en consideración en un juicio o razonamiento. La idea de un mundo del pensamiento, en que los conceptos pueden tener un valor lógico distinto de otro mundo o sistema, parece dar a la verdad una base convencional y relativa. Una proposición, dicen, puede ser verdadera en el mundo del pensamiento de la Zoología, como ningún elefante vuela, y puede no serlo en el mundo de la fábula. De forma que un mundo del pensamiento puede valer para el sujeto o grupo de inteligencias que viven en el mismo ambiente intelectual, pero carecerá de sentido para los demás.

Por lo tanto, aunque habitamos un solo Universo localizado podemos crear universos paralelos no localizados fruto de nuestros pensamientos y esto es lo que explica que la realidad de dos personas pueda ser muy diferente compartiendo el mismo espacio geográfico.

La Geomediación trata de llegar a acuerdos mediante el establecimiento de asociaciones entre universos paralelos para que se produzca un entendimiento entre distintas realidades y así evitar el caos. Supongamos que un cierto número de personas en sus respectivos coches se aproxima a unos semáforos: si no estuviesen de acuerdo unas con otras en lo que es la luz y el color, no cabe duda que se produciría un accidente catastrófico. El hecho es, sin embargo, que todos esos conductores experimentan el mismo conjunto de impresiones sensibles, que atribuyen a la existencia objetiva de una luz roja y se paran, o una luz verde y siguen su camino. En este caso los conductores por medio de asociaciones aprenden a detectar las relaciones existentes entre los acontecimientos que ocurren en su entorno y aprenden a detectar las consecuencias que tiene su comportamiento.

\section{La Geomediación desde el Sur de Europa}

La definición de la idea de Geomediación requiere del conocimiento del trabajo realizado por el Grupo de trabajo de Geomediación de la Asociación Alemana para la Geografía Aplicada, uno de cuyos centros radica en la Universidad de Bonn. Dentro de este grupo destacan los nombres de Barbara Schmidt-Belz, Claus Rinner, Thomas F. Gordon, Dirk Schmidt y Oliver Märker. Este último, licenciado en geografía, psicología y sociología en la Universidad de Bonn y doctor por la Universidad de Oldenburgo, realizó su tesis sobre «La mediación online como un instrumento para una planificación urbana y regional sostenible»; en ella presenta un marco para realizar participación pública basada en Internet en planificación urbana y regional sobre la base de teorías de planificación, investigación en mediación y casos de estudio.

Concretamente, la idea de geomediación se circunscribe dentro de la Mediación Geográfica que se define como la mediación aplicada a la planificación espacial. Mediación significa arbitrar en grupo la toma de decisiones a través de una tercera parte neutral, el 
mediador, quien controla los procesos de discusión, pero también concilia activamente cuando los conflictos ocurren. La Mediación Geográfica es una discusión moderada en procedimientos de planificación espacial y, por lo tanto, la Geomediación son los sistemas de soporte a los procedimientos de mediación en planificación geográfica. Puede ser una parte de los procesos de la Cooperativa de toma de decisiones espaciales y puede plantear requerimientos específicos en el uso de mapas online.

La toma colaborativa de decisiones espaciales (CSDM: Collaborative Spatial DecisiónMaking) es un campo de investigación fruto de la intersección de la Ciencia de la Información Geográfica y el trabajo colaborativo de soporte informático (CSCW: Computer-Supported Cooperative Work), y está muy relacionada con la negociación en planificación espacial.

Como ejemplo práctico, el Grupo de trabajo de Geomediación de la Asociación Alemana para la Geografía Aplicada ha lanzando como experiencia piloto el proyecto «Geomed» (Sistema de Mediación Geográfica) que suministra un soporte basado en Internet para la planificación espacial y la toma de decisiones, tanto en planificación urbana como regional. Geomed integra servicios en un soporte para la cooperación, la negociación y la cartografía online o Internet mapping ${ }^{3}$. Sus tres componentes centrales son un espacio virtual de trabajo compartido, un visor de SIG y foro de discusión para facilitar la planificación espacial y la toma de decisiones. En el foro de discusión puede participar un «mediador humano», denominándose «discusión informática mediatizada». (Figura 2)

En el proyecto piloto, que finalizó en el año 1998, participaron cinco socios en su desarrollo y cuatro socios usuarios de la ciudad de Bonn (Alemania), la ciudad de Tilburg (Países Bajos), la región de Toscana (Italia) y la Cámara Técnica de Grecia.

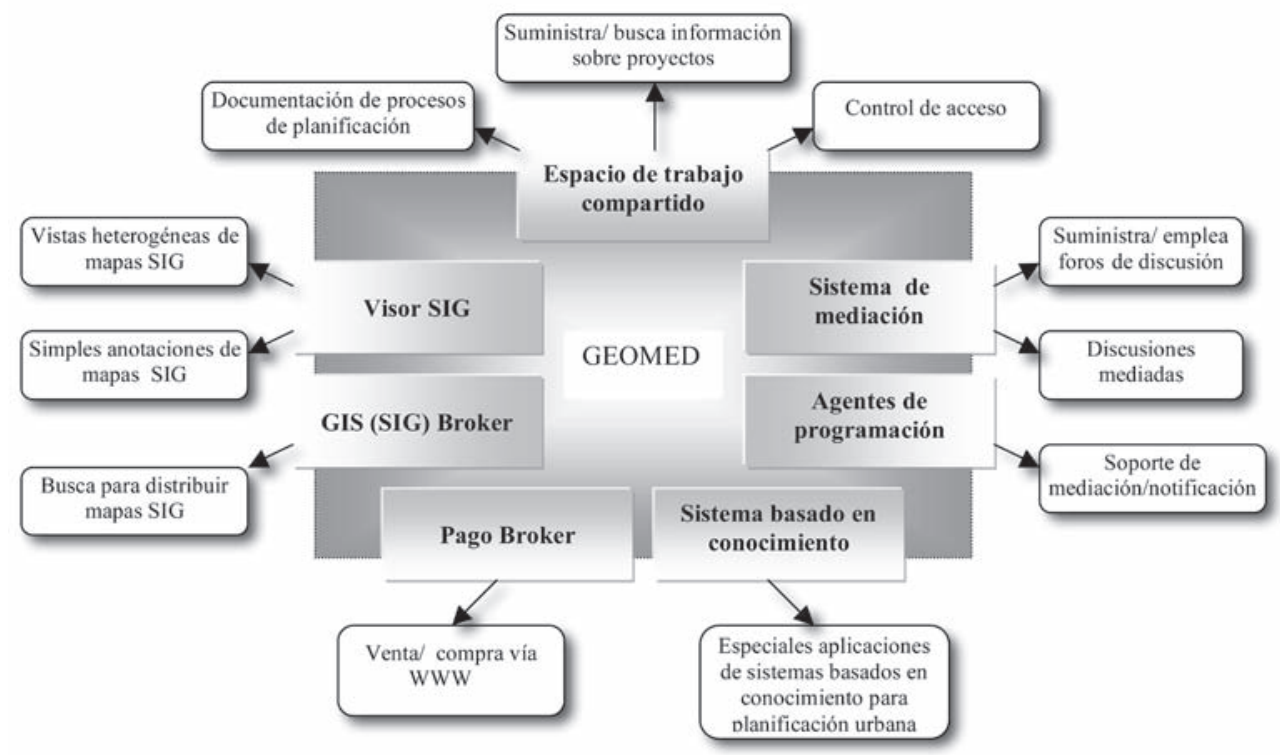

Figura 2: Proyecto Geomed. Traducción propia a partir del esquema de Schmidt-Belz, B., Rinner, C., y F. Gordon, T. (1998).

3 «Internet mapping» comprende un par de aproximaciones al trabajo con información SIG en Internet, entre las cuales los «interactive map servers» son la aplicación más extendida hoy en día. 
El término original de Geomediación en lengua alemana es «geovermittlung» aunque se emplea de forma generalizada en inglés con la expresión de «geomediation». En España todavía no se ha encontrado información especifica sobre Geomediación, quizá porque ninguna ciudad, región o entidad administrativa ha sido seleccionada para participar en el proyecto europeo Geomed y como los mismos responsables del proyecto indican la introducción de un medio innovador como Geomed dentro de la realidad de la planificación urbana y regional es un proceso lento y difícil. Sin embargo, si existen aproximaciones desde el Derecho, la Psicología o las Ciencias Sociales — especialmente en Cataluña-, más cercanas a la Mediación Geográfica. La Mediación Geográfica probablemente se encuentre con mayor difusión en España que la Geomediación ya que no se ha hallado aún información de esta idea ni en castellano, ni en otra lengua peninsular.

Lo más cercano que se ha descubierto en lengua castellana procede de la traducción de un libro en alemán llamado «Mediación en línea. Nuevos medios en la resolución de conflictos; con ejemplos de política e industria». Este libro escrito en alemán tiene un resumen en castellano que explica qué es la mediación en línea, traducido por Nicola Döring en el año 2004.

Nicola Döring nos sintetiza el libro, aclarando que la mediación en línea trata con los métodos de la resolución de conflictos que se llevan a cabo total o parcialmente a través de Internet. El libro analiza la mediación en línea como un sistema socio-técnico, a través de los significados de la plataforma tecnológica informática profundamente imbricados con la comunicación social del conflicto relatado. La primera parte del libro analiza las múltiples opciones y restricciones de la comunicación en línea para los procesos de mediación y moderación. Por ejemplo, una ventaja de la mediación en línea es que partes geográficamente separadas (p.e. en casos de comercio electrónico) pueden resolverse conflictos con un coste comparativo bajo. La segunda parte del libro plantea ejemplos de mediación en línea procedentes de la política y la industria. Aunque la mediación en línea se describe como un influyente método crecientemente solicitado, hay varios problemas socio-técnicos que necesitan abordarse en nuevas investigaciones desde este método. El libro ofrece un enfoque informático de la cuestión, centrando el tema en los países de habla alemana. Desafortunadamente, adolece de una comparación sistemática de las distintas herramientas y técnicas de mediación en línea, así como de una mirada cercana a los modelos de negocio y aspectos profesionales en éste nuevo campo de actividad.

Por la necesidad de adaptar la geomediación a cada territorio, por la falta de una referencia exacta de geomediación en lengua castellana y por el consiguiente descubrimiento que supone, se debe aportar una visión de la geomediación desde el Sur de Europa, intentando establecer las diferencias y similitudes respecto a la visión del Norte de Europa.

La Geomediación según la visión del Grupo de trabajo de Geomediación de la Asociación Alemana para la Geografía Aplicada hace referencia a los sistemas de soporte a los procedimientos de mediación en planificación geográfica. Puede ser una parte de los procesos de la Cooperativa de toma de decisiones espaciales y puede plantear requerimientos específicos en el uso de mapas online.

Desde el sur de Europa, la geomediación es un marco de intervención que engloba al conjunto de los procedimientos y/o procesos para resolver o evitar los conflictos que surgen entre los seres humanos en relación con el territorio, y favorecer la participación en la toma de decisiones sobre asuntos locales de todos los niveles de la sociedad, permitiendo potenciar los medios necesarios para la reconciliación y mejora de la sociedad, sin destruir el entorno vital. La Geomediación es un proceso que se centra en el establecimiento de asociaciones entre universos paralelos (AUPA) lo que equivaldría a lo que denominan como «toma colaborativa de decisiones espaciales» (CSDM: Collaborative Spatial Deci- 


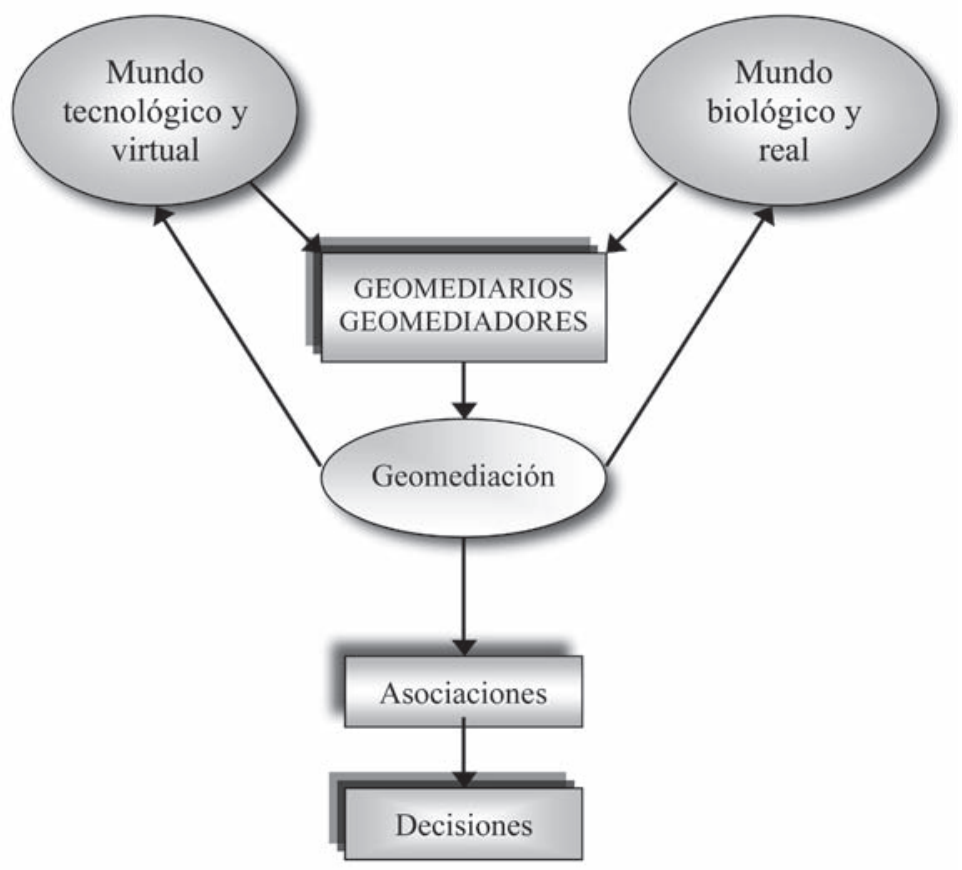

Figura 3: Proceso de geomediación. Una visión desde el Sur de Europa. Elaboración propia.

sión-Making) pero mientras allí parten de las decisiones, aquí de las asociaciones (UPAA): Associations between parallel universes). Incluso puede que el establecimiento de asociaciones sea un paso previo a la toma de decisiones (Figura 3 ).

La idea de Geomediación presenta un mayor desarrollo en Alemania, aunque ambas concepciones introducen nuevos conceptos que necesitan ser explicados.

Un punto de encuentro entre ambas concepciones puede que venga del concepto de Geomedia, ya que se conoce que existe un sistema de información geográfico (SIG) llamado Geomedia que soporta la visualización y análisis de datos en su formato nativo, los formatos estándares de imágenes y la mezcla de datos de distintas fuentes, en un marco de trabajo único e integrado. En este SIG se ha combinado lo fácil de las aplicaciones de sobremesa con el poder de las aplicaciones corporativas, creando la figura del «Cliente Geográfico Universal» una aplicación cliente que parece y se comporta como una aplicación de PC y que puede integrar datos espaciales corporativos en forma eficiente y transparente.

La geomediación no es un proceso que pueda realizar un SIG, ni los geomediarios son quienes emplean un SIG aunque hay un centro italiano que ha desarrollado un «geomediator» (geomediador) como una solución inteligente (middleware) que permite compartir geoinformación y la aplicación cooperativa. Exactamente el X-Geomediador es una herramienta práctica para acceder, visualizar y preguntar de acuerdo a los criterios de búsqueda — ¿Quién?, ¿Dónde?, ¿Cuándo?, ¿Qué?-, sobre el contenido de fuentes heterogéneas de geoinformación en Internet como si fuera un único y uniforme sistema de información local y sin modificar las bases de datos existentes. Se recomienda su uso como un posible marco en aplicaciones de control de riesgos ambientales, control de redes ambientales, sistemas de soporte de decisiones ambientales, etc. 
Además de los SIG, otro punto en común es que la geomediación procede de la combinación de geografía con mediación. Así, la geomediación puede incorporar además de conocimientos tecnológicos y geográficos, conocimientos de ecología, de sociología, de economía o incluso de humanidades y antropología, entre otros. De este modo la geomediación entra dentro de los procesos de mediación encaminados a la solución de conflictos y superación de dificultades, así como a proporcionar un foro para la toma de decisiones. Al igual que la mediación la lógica de la geomediación es ternaria y, consecuentemente, abierta, motivo por el cual permite la circulación de variables múltiples. La geomediación tiene lugar en la Tierra que no es un espacio unidimensional, ni bidimensional, sino tridimensional, voluminoso, con multiplicidad de caras, ángulos, superficies y texturas siempre variables bajo la mirada de cada uno de los participantes, sensible al paso del tiempo.

La geomediación nace de la mediación en geografía para mejorar la eficiencia y la calidad de los procesos de planificación. Es, por lo tanto, un no-poder en el sentido de que la toma de decisiones de los geomediarios y de los geomediadores es fruto de un consenso entre ciudadanos.

El Grupo de trabajo de Geomediación de la Asociación Alemana para la Geografía Aplicada define la figuras del «mediador humano»y del «GIS broker» (SIG broker). El mediador humano actúa como una tercera parte en los grupos de discusión virtual para asistir en problemas de decisión controvertida en planificación espacial como por ejemplo identificar intereses (p.ej. mantenimiento de las condiciones de vida) desde posiciones que fueron declaradas con referencia a objetos geográficos (e.j. negativa de aceptación de una estación de energía eólica cerca de la casa de un vecino). Por su parte el SIG broker o geobroker es un agente financiero que se dedica a actuar como intermediario entre empresas y localidades, ofreciendo nuevos servicios donde la información SIG puede ser vendida y respectivamente cobrar por el uso de esa información.

Las figuras correspondientes al mediador humano y al SIG broker serían el geomediario y el geomediador, si bien la geomediación es común a ambos.

Tanto el geomediario como el geomediador son imparciales y ambos deben contar con conocimientos de geografía, pero mientras el geomediario centra su relación con el territorio en los medios en este caso tecnológicos aunque también pueden ser medios de transporte o de vida, el geomediador en los conflictos.

El geomediador trata de facilitar que los territorios encuentren soluciones desde una posición de no violencia, de respeto a la libertad de los territorios y a la dignidad así como a la autonomía de las personas. Por ejemplo, un geomediador puede intervenir en los procesos de deslocalización de actividades y de creación de empleo, riesgos y catástrofes naturales, epidemias, hambrunas, crisis energéticas, degradación ambiental, turismo masivo, etc., como un altruista que busca una relación de bienestar entre la tierra y la humanidad de modo que el bienestar general derive en bienestar individual y no al revés.

Un caso práctico en el que un geógrafo puede mediar en un conflicto de modo tan singular que supera la función del planificador y se convierte en un geomediador lo encontramos en el artículo de Oliver Märker y Dirk Schmidt sobre el sistema de Geomediación Zeno en www como plataforma de participación por una nueva cultura de la planificación (1999: 163). Ambos autores emplean un modelo argumentativo para resolver un conflicto de planificación. (Figura 4)

En este caso con ayuda de un geomediador se desarrolla la estructura de argumentación que permite la comprensión de un problema y dar a conocer las consecuencias del problema y las posibles soluciones. Un geomediador desde el diagnóstico de problemas puede encontrar las claves que permiten adecuar las actividades humanas al entorno en el que se desenvuelven. 


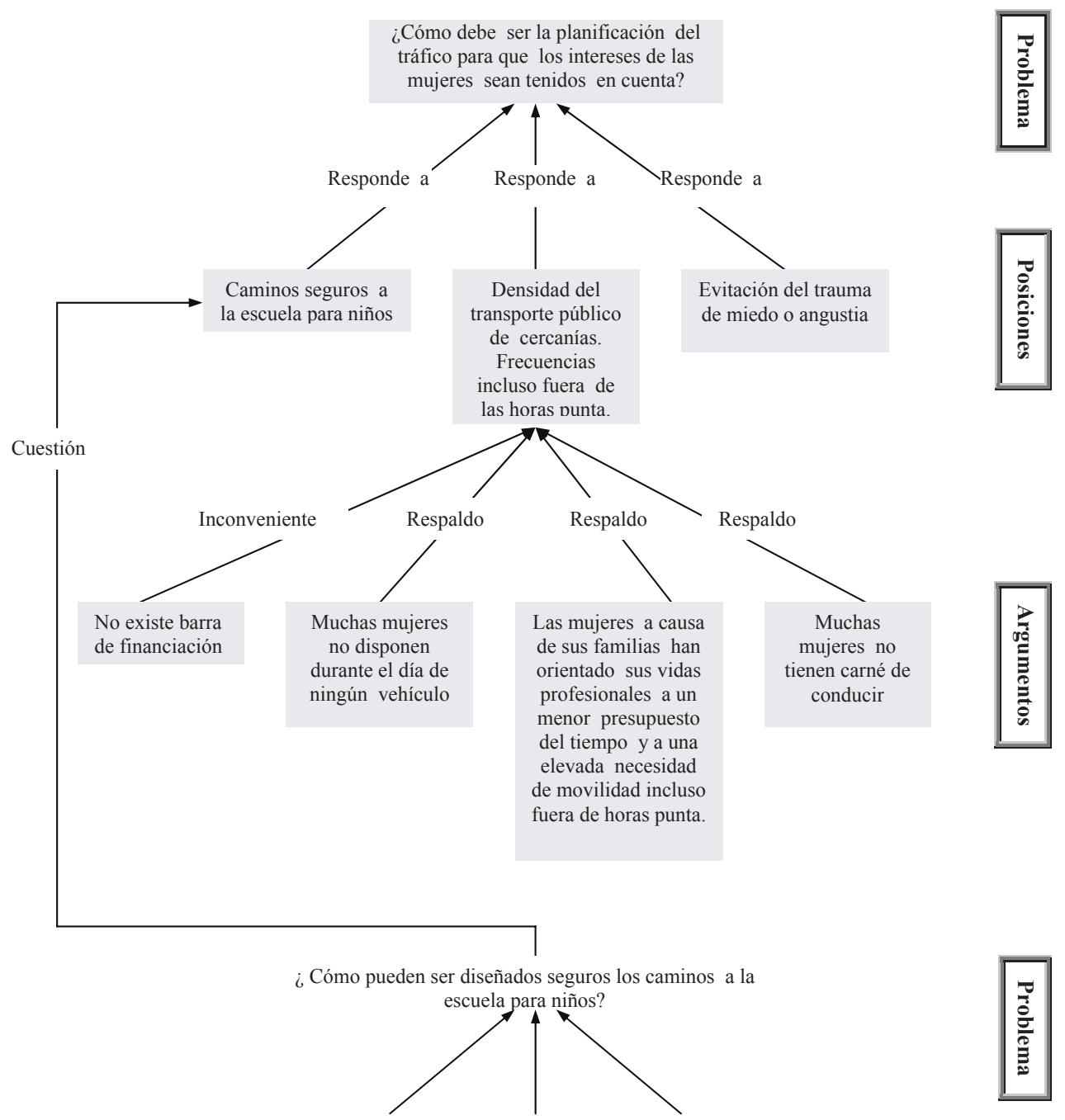

FIgURA 4: Modelo argumentativo para resolver problemas. Los principales elementos del modelo argumentativo son los problemas, las posiciones y los argumentos, estos también serán calificados como nudos. Por medio de estos nudos se desarrollará un árbol de discusión jerárquico, los nudos serán enlazados unos con otros en diferentes tipos de relaciones. Estos tipos de relaciones son como Responde a entre una posición y un problema; Respaldo entre un argumento y una posición; Inconveniente a entre un argumento y una posición; y cuestión entre un problema y una posición o entre un problema y un argumento. Para un problema básico serán formuladas preguntas. Las preguntas corresponden al nivel más alto del árbol de argumentación. La contestación a las preguntas será resultado de las diferentes posiciones o propuestas. Las posiciones o propuestas serán formuladas sobre argumentos y contra argumentos. Posiciones y argumentos pueden ser transformados en una nueva orientación del problema e introducir un nuevo árbol jerárquico planteando un nuevo problema. Traducción propia a partir del artículo de Märker, O., y Schmidt, D., (1999: 163). 
La figura de la geomediaria y de la geomediadora surge a la vez que la figura del geomediario y del geomediador. Ello quiere decir que no es una figura que encaje con un papel masculino o femenino ya preestablecido. Ambos sexos pueden desempeñar esta labor y de hecho la calidad de vida de un territorio se vincula a que haya una tendencia hacia la igualdad.

\section{Los geomediarios y las asociaciones entre universos paralelos (AUPA)}

El geomediario es un concepto nuevo que apenas ha sido empleado, aparece en un dominio de internet como www.geomediary.com pero sin conexión a una página web y en el «website» de Slashdot que pertenece al Grupo de recursos tecnológicos abiertos (OSTG). Los «websites» de OSTG proveen una única combinación de noticias, artículos originales, recursos descargables y foros comunitarios para ayudar a gerentes de Tecnología de la Información, profesionales de su desarrollo y usuarios finales a tomar decisiones críticas sobre productos y servicios de Tecnología de la Información.

El concepto del cual se deriva Geomediario es «Infomediario», pero mientras el Infomediario actúa en la red proporcionando servicios de información con fines lucrativos, el Geomediario actúa entre el mundo virtual y el mundo real, proporcionando servicios o medios de información geográfica.

Se indica que el infomediario es el verdadero protagonista del comercio electrónico; vende información valiosa sobre un mercado y crea la plataforma virtual para que vendedores y compradores puedan realizar sus negocios. Los infomediarios, de esta manera, están en condiciones de establecer las bases para la creación de nuevos mercados en torno a los

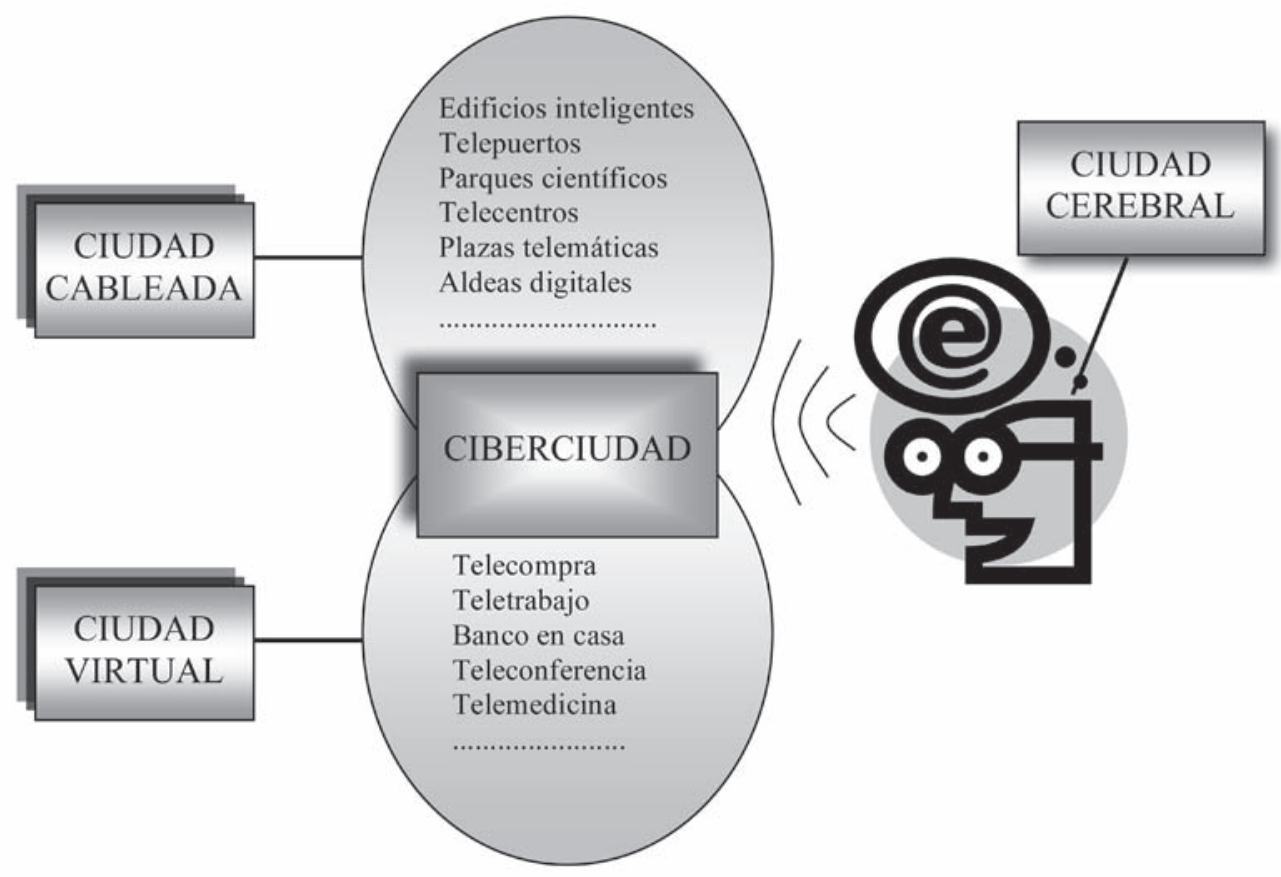

FIGURA 5: El nacimiento de la ciberciudad. Traducción propia a partir de la imagen de Romano Fistola y Rosa Anna La Rocca (1998: 195-227). 
cuales compradores y vendedores, en muchos casos desconocidos entre ellos inicialmente, establecen relaciones comerciales de interés para ambos. El infomediario presenta como valor añadido, su capacidad y habilidad para estructurar la oferta que presenta la red de forma que los consumidores los consideren como un elemento clave a la hora de llevar a cabo sus transacciones en Internet.

Con referencia al Infomediario, el Geomediario intenta optimizar la relación de la sociedad con el territorio vendiendo información geográfica y creando la plataforma virtual para que los ciudadanos puedan realizar sus gestiones respecto al territorio. Los geomediarios, de esta manera, están en condiciones de establecer las bases para mejorar la relación entre sociedades y territorios. El geomediario es capaz de estructurar los flujos de transacciones en Internet de tal forma que las oportunidades se distribuyan por el mayor número de áreas posible considerando el influjo de las tecnologías.

Algún atisbo de lo que es un geomediario lo representan Romano Fistola y Rosa Anna La Rocca (1998: 195-227). Ambos pueden ser calificados, en parte, de geomediarios ya que considerando las transformaciones que pueden causar la introducción de las redes en el sistema urbano y en función de algunos cambios que ya se están produciendo como la presencia de megacentros comerciales en los cinturones metropolitanos para atender la demanda de transacciones por Internet, proyectan el nacimiento de una nueva ciudad, la «ciberciudad». (Figura 5)

Según Romano Fistola y Rosa Anna La Rocca (1998: 195-227) la ciberciudad es originada por la intersección del conjunto de elementos caracterizados por la «ciudad cableada» y la «ciudad virtual». La ciberciudad también está determinada por el impacto psico-perceptivo que las redes ejercerán en la ciudad del futuro.

Dichos autores suponen que la introducción de la tecnología digital producirá una sensible modificación de los sistemas de composición de la ciudad, actuando primero en el sistema funcional (justicia, educación, salud, etc.) y permitiendo el acceso remoto a servicios deslocalizados.

Por consiguiente, presumen un desarrollo de un nuevo orden físico de la ciudad, caracterizado por nuevas distribuciones de las construcciones en la ciudad y por formas urbanas cada vez más parecidas a «lugares electrónicos», donde los ciudadanos convertidos en ciberciudanos expresaran demandas asociadas a un nuevo estilo de vida.

Con este planteamiento los autores intentan llamar la atención de los planificadores urbanos para que consideren las redes no sólo como una infraestructura urbana sino como un cambio en el modo de pensar la ciudad.

La geomediación tal y como se plantea en el Norte de Europa y se trata de introducir en otros países está muy centrada en la planificación urbana aunque también incluye la regional. De hecho el proyecto Geomed aspira a mejorar la planificación urbana y regional, incluyendo la participación pública. En la experiencia piloto del proyecto Geomed se marcaron como objetivos:

- hacer los procesos de planificación más transparentes,

- facilitar la participación pública,

- ayudar a evitar o resolver conflictos,

- facilitar y mejorar la cooperación entre planificadores, expertos y comunidades, y

- hacer la planificación urbana más eficiente.

En este sentido es un proceso muy vinculado al gobierno electrónico dado que el gobierno electrónico supone una liberalización de los servicios públicos a través de la adopción de las tecnologías de la comunicación y la información. El e-gobierno no consiste solamente 
en la introducción de tecnología, pretende reinventar el modo en el cual los proveedores de servicios y los clientes interactúan, transformando los procedimientos de negociación, la dirección de la comunidad, posibilitando el desarrollo económico y renovando el rol del gobierno en la sociedad.

El proceso de geomediación va más allá de las ciudades pues contempla todo el territorio incluyendo las zonas rurales, marítimas e insulares, ya que la geomediación, según se concibe, no pretende ser un transplante de sistemas urbanos existentes a un entorno virtual sino un proceso que intenta contribuir a mejorar el uso y organización del territorio, incluyendo el modo de vida y producción sobre el mismo, impidiendo a su vez que sea únicamente el flujo de transacciones el que dirige la planificación de los territorios desde una posición de no-poder. Para este propósito se deberían crear Centros de Geomediación que dispongan de independencia dado que con la geomediación se trata de rehuir de los planteamientos binarios (ciudad-pueblo, norte-sur, centro-periferia) por medio de asociaciones que contribuyan a romper la dualidad y permitan centrarse en el análisis de los fenómenos.

La Geomediación al intentar entender el mundo es un proceso que puede derivar en la creación de contextos favorables para solucionar algunos conflictos, en especial aquellos en los que interviene la calidad de vida. En este sentido puede contribuir a modificar la percepción de nosotros mismos y de nuestro entorno de modo que seamos más conscientes de la realidad del planeta y de sus posibilidades.

Las circunstancias territoriales locales que forman el hábitat son las que plantean los principales problemas de la vida y la geomediación ofrece procesos y procedimientos que ayudan a solucionarlos. El paso hacia la geomediación es decisivo para alcanzar el bienestar y por ello éstas son algunas pinceladas desde el Sur de Europa de un proceso que hay que desarrollar más en próximas investigaciones completándolo y contrarrestándolo con información de otros países y regiones, especialmente de Alemania. Es importante destacar que si bien la era de la informática y las comunicaciones está monopolizada, prácticamente, por las empresas estadounidenses, son los investigadores alemanes los que tienen la primacía del conocimiento en la sociedad de la información.

Desde el Sur de Europa la geomediación puede comenzar por la toma de contacto directo (viajes, visitas, estancias, excursiones, etc.) o indirecto (mapas, fotografías, revistas, libros, noticias, documentales, películas, imágenes aéreas o de satélite, datos estadísticos, etc.) con el territorio. A partir de la información recogida se puede reflexionar sobre las posibilidades de mejorar la calidad de vida, de relación con el territorio y la naturaleza y de expectativas de futuro desde aspectos muy concretos de fácil solución a más difíciles. El punto central de la geomediación, de los geomediarios y de los geomediadores es el establecimiento de asociaciones entre universos paralelos (AUPA) y éstas deben buscarse en el inmediato futuro, evitando repetir o recuperar el pasado si éste resultara de carácter negativo pero sin dejar de mirar atrás en cuanto a la problemática ambiental y social.

Una vez conocida la situación actual de los territorios y valorado los medios en este caso tecnológicos que pueden contribuir a mejorar la calidad de vida no sólo en tanto que crecimiento económico sino más bien en tanto que acentuación del máximo bienestar con el ínfimo consumo, se puede proceder a establecer asociaciones.

Al explorar las posibilidades, no hemos de pensar en la tecnología y la geografía como elementos estáticos, sino que debemos estar dispuestos a tener en cuenta la posibilidad de que la tecnología y geografía evolucionen o cambien e intentar que las asociaciones sirvan para que ambos elementos evolucionen o cambien al mismo ritmo.

Provisionalmente hay varias asociaciones que se mencionan mucho y pueden servirnos de referencia:

Internet como medio que puede: 
- Reducir las tensiones que sufren las áreas urbanas, al permitir a las personas trabajar desde sus hogares o desde oficinas alejadas de los centros urbanos.

- Reducir el abandono y desempleo en zonas rurales y /o aisladas al descentralizar la actividad económica en el territorio.

- Reducir las presiones sobre los recursos naturales porque un número cada día mayor de productos, adquirirán la forma de bits en lugar de la de bienes manufacturados.

- Reducir la contaminación del aire provocada por el dióxido de carbono procedente de la combustión de derivados del petróleo al disminuir el número de desplazamientos.

Existen métodos sobre aprendizaje asociativo que ayudan al conocimiento sobre el proceso de establecer asociaciones. En éstos métodos se indica que en un proceso de asociacionismo intervienen cuatro elementos: Estímulo Incondicionado (EI), Respuesta Incondicionada (RI), Estímulo Condicionado (EC), y Respuesta Condicionada (RC). De forma breve se explica que conociendo previamente la RI, el EC actúa sobre el EI dando lugar a una RC de tal manera que se establece la asociación. Por ejemplo, en tiempos pasados muchos barcos naufragaban cerca de las costas porque se encallaban en las rocas al no poder divisar la costa. Éste hecho motivo la construcción de faros para advertir de la presencia de la tierra y así disminuyó el número de hundimientos. En éste ejemplo la RI es el número de naufragios, el EC son los faros, el EI el mar y la RC la reducción del número de naufragios. Con dicho ejemplo se manifiesta que la fusión creadora de la tecnología y la geografía es muy poderosa pero conviene dotar a la geomediación de un carácter equilibrado entre la aplicación de tecnologías y los valores biológicos y humanos para evitar caer en el nihilismo. Ya hay ciertos tipos de empleos y actividades que son superfluos debido a las nuevas tecnologías, asimismo sin el conocimiento previo y fundamental sobre el estado y sentido de la vida en la Tierra, la geomediación acabaría siendo innecesaria y sus resultados no tendrían compensación.

Basta recordar que la fundación Greenpeace es pionera en la implantación de la mediación para resolver conflictos ambientales.

La Geomediación se configura como una experiencia de profesionalización de la Geografía y por este motivo del desarrollo de la capacidad o del interés del geógrafo y de otros interesados por la geomediación se deriva parte de su potencial como profesional y académico, ya que es un proceso que tiene un carácter sobre todo propositivo. Es recomendable que en un proceso de geomediación intervengan varios geomediarios y geomediadores porque así se pueden enfocar las asociaciones desde perspectivas diferentes o bien conciliar posturas que es uno de los mayores problemas del mundo. Además, si bien es importante definir la figura de un geomediario y un geomediador igualmente lo es al referirse en plural como geomediarios y geomediadores ya que es necesario conseguir una igualdad incluso dentro de la geomediación y de ésta forma se puede expandir la idea para que sea una realidad compartida.

\section{Bibliografía:}

FISTOLA, R. Y LA ROCCA, R.A.(1998): «Cybercities: a new way of thinking about the town planning of the future - the case of Naples-》. En BAKIS H. Y SEGUI, J.M (Editores): Netcom, Volume 12, no 1-2-3, pp. 195-227.

FRAIOLI, L. (1999): La Historia de la tecnología. Editorial Editex, Italia, pp. 124.

MÄRKER, O., Y SCHMIDT, D. (1999): «Zeno - GeoMediationssystem im WWW - Potentiale von Issue Based Information Systems (IBIS) als Beteiligungsplattform einer neuen PlanungsKultur». 
En SCHRENK M: Symposion zur Rolle der Informationstechnologie in der Raumpung. pp. 161171.

RINNER, C.: Online maps in Geomed. Internet Mapping, online GIS and their aplicattion in Collaborative Spatial Decision-Making. German National Research Center for Information Tecnology.

SCHMIDT-BELZ, B., RINNER, C. Y F. GORDON T. (1998): GeoMed for Urban Planning. First User Experiences. German National Research Center for Information Technology.

VINYAMATA, E.(2003): Aprender mediación. Editorial Paidos. Barcelona, pp. 